\title{
Spontaneous closure of stage two idiopathic macular hole with persistent vitreous attachment
}

\author{
Rashed Mustafa Nazzal@, Dominika Wrzesińska®, Katarzyna Nowomiejska®, Robert Rejdak® \\ Department of General Ophthalmology, Medical University of Lublin, Lublin, Poland
}

\begin{abstract}
The classical rule was so far to observe idiopathic macular holes of stage $1 \mathrm{a}$ and $1 \mathrm{~b}$ because of the high probability of spontaneous closure of the hole, while in higher stages surgical treatment was recommended. However, a growing number of case reports are available nowadays that suggest that even a more advanced stage macular hole may occasionally close spontaneously. In this article, we present a case of a 71-year-old female patient who presented with gradual bilateral decrease of vision, which was attributed to a combination of cataract and macular holes in both eyes. The macular hole in one eye was stage $1 \mathrm{~A}$ and the other eye was stage 2 . Follow up over a period of 6 months revealed conversion of the stage $1 \mathrm{~A}$ hole to lamellar hole and spontaneous closure of the stage 2 hole.
\end{abstract}

KEY WORDS: idiopathic macular hole; spontaneous closure; optical coherence tomography

Ophthalmol J 2019; Vol. 4, 49-51

\section{INTRODUCTION}

A full-thickness macular hole is defined as complete loss of tissue from the foveal center; it can be traumatic or more commonly, idiopathic. It has been classically staged by Gass into four categories: in stage 1 a yellow spot or halo develops associated with loss of the normal anatomic foveal depression. No vitreous separation is present. This may resolve or progress to a small (less than 400 micron), early macular hole (stage 2). This hole gradually enlarges to a diameter more than 400 micron. The vitreous usually remains attached (stage 3). Some eyes have complete posterior vitreous separation (stage 4) [1]. The spontaneous closure of idiopathic macular hole is not new; it has been documented many years ago, even before the introduction of vitrectomy as a standard way of managing this entity. ${ }^{2}$

\section{CASE REPORT}

A 71-year-old female patient presented with gradual painless decrease of vision in both eyes for the past several months. On examination, best corrected visual acuity was 0.2 in the right eye and 0.1 in the left eye. Anterior segment examination showed cataract nuclear sclerosis of +3 in each eye. Optical coherence tomography (OCT) revealed stage $1 \mathrm{~A}$ macular hole in the right eye and full-thickness macular hole stage 2 (290 microns of minimal hole width) with persistent vitreous attachment in the left eye (Fig. 1). Patient was given an appointment for combined cataract extraction and vitrectomy in the left eye after 6 months. When she came back for the surgery, she reported mild improvement of vision in the left eye. OCT was done again and revealed spontaneous closure of the macular hole in the left eye and conversion from 


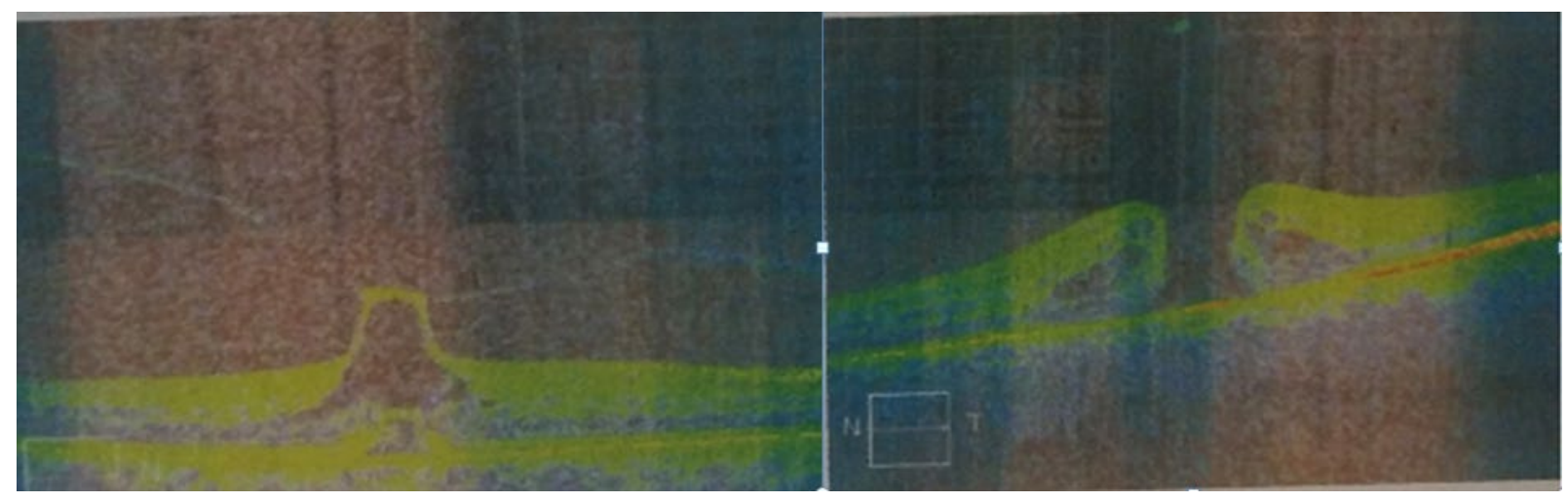

FIGURE 1. Optical coherence tomography of both eyes at presentation (left — right eye, stage 1A macular hole, right — left eye stage 2 macular hole)

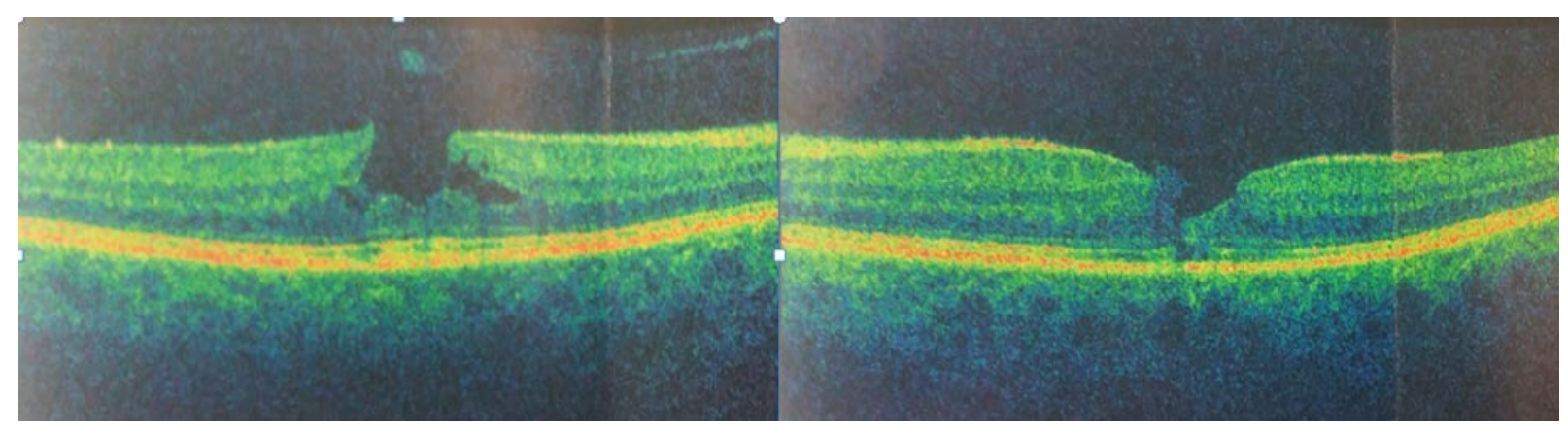

FIGURE 2. Optical coherence tomography of both eyes after 6 months (left — right eye, resolution into lamellar macular hole; right - left eye, closed macular hole)

1A macular hole to lamellar hole in the right eye (Fig. 2). The vision remained 0.2 in the right eye but improved to 0.3 in the left eye.

\section{DISCUSSION}

The proportion of spontaneous closure in idiopathic macular hole has been reported to be 2.7-6.2\% [2-4]. However, relatively higher proportion $(10.7 \%$ to $44.4 \%)$ of traumatic macular holes close spontaneously. Therefore, an observation for a period of up to four months may be a management of choice for traumatic macular hole [5].

The mechanism of macular hole closure or the predictive factors for possible spontaneous closure is not understood very well and most of our assumptions came from previous studies about traumatic macular holes; for example, a study was published by Haoyu Chen et al. identified two predictive factors associated with spontaneous closure of traumatic macular holes. The first factor is small minimum diameter of the macular hole at baseline, which may allow easy migration of glial cells. The other associ- ated factor was absence of intraretinal cysts, which may be an indicator of internal limiting membrane traction, which may activate Müller cells and cause accumulation of extracellular fluid in the retina [6]. Further studies are needed to establish whether or not these assumptions can be extrapolated to idiopathic age-related holes.

Some explanations have been proposed for the spontaneous resolution of a macular hole. These include: complete detachment of the posterior hyaloid from the foveal area leading to a release of traction; cell proliferation at the base of the hole; formation of a contractile epiretinal membrane resulting in shrinkage and closure of the hole; and bridging of the retinal tissue across the hole [7].

Although these explanations may sound highly convincing, a number of case reports show contradictions to at least some of them - such as our case in which the vitreous is still attached as well as case reports by Kelkar et al. [8] and Sugiyama et al. [3].

Since OCT revealed the closure of the macular hole with the resolution of the cystic spaces and bridging of the inner retinal layers with or without 
lamellar defects and without any posterior vitreous detachment or glial tissue proliferation, it is thought that the bridging of the retinal tissue allowed the resolution of the cystoid spaces by preventing the influx of vitreous fluid into intraretinal spaces and therefore leading to a spontaneous closure of the macular hole [8].

Hence, out of the 4 mechanisms mentioned above, posterior vitreous detachment and epiretinal membrane formation may or may not always be evident in patients with spontaneous closure of a macular hole, but the bridging of the sensory retina and the smaller size of the macular hole appear to be the most consistently reported findings for the spontaneous closure of macular holes [9].

As to the origin of the bridging of the retinal tissue, proliferation of the retinal post-mitotic neurosensory cells has been proposed, but could not be identified; proliferation of glial or retinal pigment epithelial cells has also been suggested. Since the concept of cell proliferation as a mechanism of macular hole closure is still speculative, the exact mechanism of how the spontaneous macular hole closure with maintained normal retinal structure occurs is still unclear [10].

\section{CONCLUSIONS}

Although spontaneous closure is well known in traumatic macular hole, this phenomenon is less well recognized in idiopathic age-related macular hole. The growing number of case reports of spontaneous closure may promote changing in our way to approach these cases; which means that a waiting period of few months before surgery may be warranted for selected cases.

\section{ACKNOWLEDGEMENTS}

None.

\section{CONFLICT OF INTERESTS}

None.

\section{REFERENCES}

1. Johnson RN, Gass JD. Idiopathic macular holes. Observations, stages of formation, and implications for surgical intervention. Ophthalmology. 1988; 95(7): 917-924, indexed in Pubmed: 3174041.

2. Yuzawa $M$, Watanabe $A$, Takahashi $Y$, et al. Observation of idiopathic full-thickness macular holes. Follow-up observation. Arch Ophthalmol. 1994; 112(8): 1051-1056, doi: 10.1001/archopht.1994.01090200057022, indexed in Pubmed: 8053818.

3. Sugiyama A, Imasawa M, Chiba T, et al. Reappraisal of spontaneous closure rate of idiopathic full-thickness macular holes. Open Ophthalmol J. 2012; 6: 73-74, doi: 10.2174/1874364101206010073, indexed in Pubmed: 22934124.

4. Privat $E$, Tadayoni $R$, Gaucher $D$, et al. Residual defect in the foveal photoreceptor layer detected by optical coherence tomography in eyes with spontaneously closed macular holes. Am J Ophthalmol. 2007; 143(5): 814-819, doi: 10.1016/j.ajo.2006.12.039, indexed in Pubmed: 17362861.

5. Yamashita $T$, Uemara A, Uchino E, et al. Spontaneous closure of traumatic macular hole. Am J Ophthalmol. 2002; 133(2): 230-235, doi: 10.1016/s0002-9394(01)01303-4, indexed in Pubmed: 11812427.

6. Chen H, Chen W, Zheng K, et al. Prediction of spontaneous closure of traumatic macular hole with spectral domain optical coherence tomography. Sci Rep. 2015; 5: 12343, doi: 10.1038/srep12343, indexed in Pubmed: 26196460.

7. Menchini U, Virgili G, Giacomelli G, et al. Mechanism of spontaneous closure of traumatic macular hole: OCT study of one case. Retina. 2003; 23(1): 104-106, indexed in Pubmed: 12652242.

8. Kelkar AS, Bhanushali DR, Kelkar JA, et al. Spontaneous Closure of a Full-Thickness Stage 2 Idiopathic Macular Hole without Posterior Vitreous Detachment. Case Rep Ophthalmol. 2013; 4(3): 188-191, doi: 10.1159/000356125, indexed in Pubmed: 24348401.

9. Kim JW, Freeman WR, el-Haig W, et al. Baseline characteristics, natural history, and risk factors to progression in eyes with stage 2 macular holes. Results from a prospective randomized clinical trial. Vitrectomy for Macular Hole Study Group. Ophthalmology. 1995; 102(12): 1818-1828, indexed in Pubmed: 9098283.

10. Guyer DR, Green WR, de Bustros $S$, et al. Histopathologic features of idiopathic macular holes and cysts. Ophthalmology. 1990; 97(8): 1045-1051, indexed in Pubmed: 2402416. 